\title{
Desempenho ocupacional de idosos praticantes de hidroginástica
}

\author{
Kátine Marchezan Estivalet ${ }^{\mathrm{a}}$, Sara Teresinha Corazza ${ }^{\mathrm{b}}$ \\ ${ }^{a}$ Departamento de Terapia Ocupacional, Universidade Federal de Santa Maria - UFSM, Santa Maria, RS, Brasil. \\ bDepartamento de Métodos e Técnicas Desportivas, Universidade Federal de Santa Maria - UFSM, \\ Santa Maria, RS, Brasil.
}

\begin{abstract}
Resumo: Introdução: Sabe-se que a prática de hidroginástica tem efeitos positivos na saúde, contribuindo para um envelhecimento ativo. Entretanto, considerando as alterações decorrentes do processo de envelhecimento, o idoso pode apresentar dificuldade na realização de atividades cotidianas, indicando problemas no desempenho ocupacional. Objetivo: Identificar as atividades-problema no desempenho ocupacional, além de correlacionar o desempenho e a satisfação de idosos praticantes de hidroginástica com o seu desempenho ocupacional. Método: Estudo transversal, do tipo descritivo correlacional, realizado com 45 idosos participantes de um projeto de extensão de aulas de hidroginástica. Foi aplicado um questionário para caracterização sociodemográfica e foi realizada avaliação do desempenho através da Medida Canadense de Desempenho Ocupacional. Resultados: A área com maior número de idosos com problemas no desempenho ocupacional e também por envolver as atividades consideradas mais importantes, principalmente a prática de outro exercício físico, foi o lazer. Entre os principais problemas de desempenho ocupacional, sete atividades estão relacionadas ao lazer, oito à produtividade e nove ao autocuidado - principalmente pela dificuldade na mobilidade funcional. Houve uma correlação significativa entre o desempenho e a satisfação dos idosos $(r=0,770$ e $\mathrm{p} \geq 0,001)$. Conclusão: Idosos praticantes de hidroginástica têm problemas no desempenho ocupacional. Assim, considera-se importante a execução de projetos com propostas de outros exercícios físicos, uma vez que são consideradas atividades importantes para os idosos, contribuindo para um envelhecimento ativo e com qualidade de vida na velhice, refletindo no desempenho positivo de atividades de autocuidado e de produtividade.
\end{abstract}

Palavras-chave: Idoso, Desempenho Ocupacional, Atividade Física.

\section{Occupational performance of elderly practicioners of water aerobics}

\begin{abstract}
Introduction: The practice of water aerobics has acknowledged positive effects on health, contributing to an active aging. However, considering the alterations brought about by the aging process, the elderly may present difficulties to perform daily activities, indicating problems in occupational performance. Objective: To identify the problem-activities in occupational performance of the elderly who practice water aerobics and correlate their performance and satisfaction with their occupational performance. Method: Cross-sectional, correlational and descriptive study conducted with 45 elderly enrolled in an outreach project of water aerobics classes. A questionnaire was applied for sociodemographic characterization and an occupational performance evaluation was performed through the Canadian Measure of Occupational Performance. Results: Leisure was the area in which the highest number of elderly people has occupational performance problems. This area also involves activities considered more important by the elderly, mainly the practice of other physical exercises. Among the main occupational performance problems, seven activities are related to leisure, eight to productivity and nine to self-care - mainly due to the
\end{abstract}

Autor para correspondência: Kátine Marchezan Estivalet, Centro de Educação Física e Desportos, Universidade Federal de Santa Maria, Av. Roraima, 1000, Prédio 51, Sala 1021, Bairro Camobi, Cidade Universitária, CEP 97105-900, Santa Maria, RS, Brasil, e-mail: katinemarchezan@gmail.com Recebido em Set. 23, 2016; $1^{\text {a }}$ Revisão em Dez. 20, 2016; Aceito em Fev. 21, 2017. 
difficulty in functional mobility. There was a significant correlation between performance and satisfaction of the elderly ( $r=0.770$ and $p \geq 0.001$ ). Conclusion: Elderly practitioners of water aerobics have problems in occupational performance. Thus, the development of projects with proposals of other physical exercises is considered fundamental. Such activities are important for the elderly, as they contribute to an active aging and to the quality of life in old age, reflecting the positive performance of self-care activities and productivity.

Keywords: Elderly, Occupational Performance, Physical Activity.

\section{Introdução}

No processo normal de envelhecimento, também denominado de senescência, são comuns alteraçóes orgânicas, podendo ocasionar perdas de saúde funcional e fisiológica, além da possibilidade de estar relacionado também com a presença de doenças crônicas, denominando-se de senilidade ou envelhecimento patológico (FECHINE; TROMPIERI, 2012). Diante do processo de envelhecimento, normal ou não, há situaçôes que vão interferir diretamente no cotidiano dos idosos, podendo acarretar alguma limitação nas atividades de vida diária, com um aumento do risco de se tornar dependente (LANGELAND et al., 2015).

O desempenho ocupacional, foco de intervenção da terapia ocupacional, é o resultado das interaçôes entre a pessoa, o ambiente e a ocupação (PEDRETTI; EARLY, 2004). O desempenho ocupacional trata-se da participação integrada e equilibrada do indivíduo na realização de suas atividades cotidianas em três áreas essenciais: autocuidado, produtividade e lazer, sendo determinado pelo próprio indivíduo com base em suas experiências, pois possibilita determinar suas habilidades, bem como a satisfação para desempenhar as suas ocupaçôes cotidianas (LAW et al., 2009).

Especialmente entre as pessoas acima de 60 anos de idade, considera-se o exercício físico um dos principais fatores na relação saúde-doença e na melhoria da qualidade de vida (ZAGO, 2010). O exercício em meio líquido, ou aquático, como a hidroginástica, é uma opção positiva para a população idosa (SILVA; RIBEIRO, 2010), em vista do emagrecimento geral, fortalecimento e resistência muscular, condicionamento físico geral, melhora da flexibilidade, melhora do equilíbrio e coordenação motora geral (MAZO; LOPES; BENEDETTI, 2004).

Diante dos benefícios da hidroginástica na saúde do idoso, pode-se mencionar que ela também tem efeitos positivos nos componentes do desempenho ocupacional. Assim, no componente sensório-motor, que envolve funçáo sensorial, musculoesquelética e motora; no componente dos aspectos cognitivos, principalmente as funções cerebrais superiores; e também no componente dos fatores psicossociais, abrangendo a integração social e processamento emocional
(PEDRETTI; EARLY, 2004). Por consequência, há possibilidade dos efeitos positivos da hidroginástica contribuírem nas áreas do desempenho ocupacional, podendo o idoso apresentar menor dificuldade na realização das atividades de cuidados pessoais, lazer e atividades produtivas e de trabalho.

O envelhecimento ativo trata-se do processo de otimização das oportunidades de saúde, na participação social e com segurança, sendo a prática de atividades físicas um de seus fatores comportamentais determinantes (ORGANIZAÇÃO..., 2005). Percebe-se, então, que a prática de exercícios físicos, como a hidroginástica, também tem relação com o envelhecimento, pois o estilo de vida ativo pode promover a manutenção do desempenho ocupacional dos idosos por um período mais longo, uma vez que o objetivo do envelhecimento ativo é aumentar a expectativa de vida saudável e a qualidade de vida (ORGANIZAÇÃO..., 2005). Mesmo o idoso sendo ativo, torna-se importante entender o impacto do envelhecimento no desempenho ocupacional para a realização de atividades desejadas e esperadas com satisfaçáo nas diferentes áreas ocupacionais, pois um desempenho ocupacional satisfatório permitirá ao idoso ter uma vida ativa e com qualidade.

Assim, a escolha pelo estudo parte do entendimento de que as atividades humanas estão em três áreas da vida: autocuidado, produtiva e lazer, sendo que as alteraçôes decorrentes do envelhecimento podem ser responsáveis por mudanças transitórias e também permanentes no desempenho ocupacional. Contudo, a partir do conhecimento de que a prática regular de exercícios físicos possibilita um envelhecimento ativo, o objetivo do estudo foi descrever os principais problemas no desempenho ocupacional nas áreas da ocupação, além de correlacionar o desempenho e a satisfação de idosos praticantes de hidroginástica.

\section{Método}

O estudo caracteriza-se como quantitativo, transversal, do tipo descritivo correlacional (THOMAS; NELSON; SILVERMAN, 2012). A partir dos critérios de inclusão e exclusão, e do interesse em participar da pesquisa, houve a seleção de 45 idosos 
participantes do "Projeto de Extensão Atividades Físicas da Terceira Idade - Hidroginástica" do Centro de Educaçáo Física e Desportos da Universidade Federal de Santa Maria (UFSM), no período de agosto a dezembro de 2015 .

Para a triagem e seleção dos participantes da pesquisa, houve a identificação dos matriculados no projeto, acompanhamento das aulas e controle da frequência destes nas aulas de hidroginástica. Ao término das aulas de hidroginástica relativas ao segundo semestre de 2015, os participantes incluídos na pesquisa foram avaliados quanto à condição cognitiva e responderam a uma entrevista inicial elaborada para a própria pesquisa. Após a seleção dos participantes, houve agendamento individual de cada um para realização da avaliação do desempenho ocupacional, nos meses de janeiro e fevereiro de 2016. Ressalta-se que todos os procedimentos da pesquisa, desde a triagem, seleção dos participantes e a avalição, foram realizados pela autora, terapeuta ocupacional, uma vez que o presente estudo era parte de uma dissertaçáo de mestrado do Programa de Pós-Graduação em Gerontologia da Universidade Federal de Santa Maria.

A população participante do estudo foi composta por idosos, de ambos os sexos, com idade igual ou superior a 60 anos, participantes efetivos do projeto de extensão por no mínimo um semestre, com realização apenas de exercícios físicos em meio líquido (hidroginástica), com participação mínima de duas vezes na semana e com frequência igual ou superior a $75 \%$ das aulas. No estudo, foram excluídos idosos com comprometimento cognitivo, através do Miniexame do Estado Mental (MEEM), de acordo com a escolaridade (BRUCKI et al., 2003), bem como apresentando dificuldade de comunicação, tanto de expressão quanto de compreensão, impossibilitando responder os questionamentos. Cabe ressaltar que houve a identificação das condiçóes clínicas dos participantes, por meio da entrevista inicial. Porém, mesmo os idosos com alguma doença crônica não foram excluídos do estudo, sendo estes independentes e autônomos.

A Figura 1 representa o fluxograma da seleção dos participantes do estudo, sendo que na pré-seleção houve uma exclusão inicial de 237 pessoas em função da idade inferior a 60 anos e por praticar hidroginástica apenas uma vez na semana. $\mathrm{Na}$ continuidade da pesquisa, 41 participantes foram excluídos por praticar outro exercício físico, sendo mencionadas a prática de musculação, ginástica, pilates e caminhada; 29 pelo número de faltas e 22 pela pontuação no MEEM abaixo do escore indicado pela escolaridade. Assim, foram selecionados para o estudo o total de 45 participantes.

Para investigar o desempenho ocupacional, usou-se a Medida Canadense de Desempenho Ocupacional (COPM), que se baseia na prática centrada no indivíduo, através de uma entrevista semiestruturada (LAW et al., 1990). Na aplicação da COPM, o participante precisa citar as atividades que são mais importantes no cotidiano, mas que encontra dificuldade para realizar, atribuindo uma pontuação entre 1 e 10 pontos, de forma crescente. Assim, solicitou-se a cada participante pensar em um dia comum e identificar problemas de desempenho nas seguintes áreas: autocuidado (cuidados pessoais, mobilidade funcional e independência fora de casa), produtividade (trabalho, tarefas domésticas) e lazer (recreação tranquila, recreação ativa, socialização) (MCCOLL et al., 2005).

Após a citação de todas as atividades, pontuaram-se as cinco principais (maior pontuação) com problemas de desempenho ocupacional. Em seguida, o participante autoavaliava o desempenho e a satisfação. Assim, a COPM apresenta escore de desempenho entre 1 e 10 pontos, sendo o escore 1 entendido como "incapaz de fazer" e o escore 10 como "capaz de fazer extremamente bem". O mesmo parâmetro segue para o escore de satisfação: escore 1 "nada satisfeito" e 10 pontos "extremamente satisfeito" (CUP et al., 2003). Cabe ressaltar que a COPM envolve a percepção do indivíduo quanto aos problemas de desempenho ocupacional, assim, para o estudo, a ocupação de cada participante foi categorizada nas áreas de desempenho ocupacional de acordo com seu interesse e sua necessidade (CARSWELL et al., 2004). A aplicação da COPM teve a duração, aproximadamente, de 20 minutos a 30 minutos para cada participante. Ressalta-se que

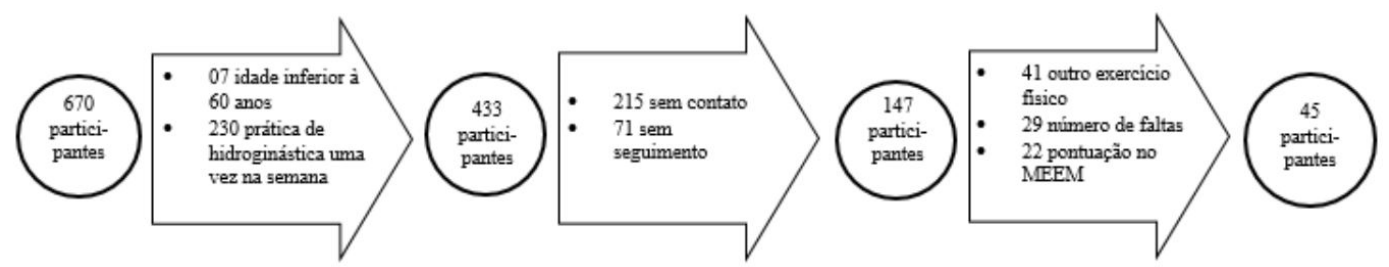

Figura 1. Fluxograma da seleção dos participantes do estudo. Fonte: Elaborado pelos autores. 
a pesquisa náo teve caráter de intervenção, sendo os escores usados apenas para efeito de descrição das principais atividades-problema e correlação da percepção entre desempenho e satisfação.

O presente estudo foi aprovado pelo Comitê de Ética e Pesquisa da Universidade Federal de Santa Maria, sob o CAEE no 45429015.4.0000.5346, e cumpre os princípios éticos conforme exigências do Ministério da Saúde, de acordo com a Resolução $n^{\circ} 466 / 2012$ (BRASIL, 2012), sendo assegurados por parte dos pesquisadores e participantes da pesquisa sigilo das informaçóes e dados coletados, bem como a resguarda destes no período de cinco anos, mediante Termo de Consentimento Livre e Esclarecido.

A análise dos dados foi realizada, primeiramente, através da descrição dos dados com média e desviopadrão. Após a verificação da distribuição normal das variáveis, foi realizada a correlaçáo de Pearson para verificar a correlação entre as variáveis: autocuidado e produtividade, autocuidado e lazer, produtividade e lazer, e desempenho e satisfação. O software usado foi o Statistical Package for the Social Sciences (SPSS), com nível de significância de 5\%.

\section{Resultados}

Para obter informações das características sociodemográficas dos participantes (Tabela 1), houve a aplicação de um questionário estruturado para os seguintes dados: idade, sexo, escolaridade, situação conjugal, religião e ocupação atual.

Após análise da Medida Canadense de Desempenho Ocupacional (COPM), cinco idosos não mencionaram nenhum problema na realização de suas atividades cotidianas. Assim, para o presente estudo, houve a consideraçáo dos resultados dos outros 40 idosos com pelo menos um problema de desempenho ocupacional.

A partir da identificação das atividades do dia a dia que são difíceis de realizar, de forma satisfatória, de acordo com as áreas do desempenho ocupacional (Tabela 2), percebe-se que a área com maior número de idosos com problemas no desempenho ocupacional está no lazer (80\%).

Houve também uma descrição das atividades-problema (Tabela 3), separadas pelas áreas de autocuidado (cuidados pessoais, mobilidade funcional e independência fora de casa), produtividade (trabalho, tarefas domésticas, brincar/escola) e lazer (recreação tranquila, recreação ativa e lazer).

Considerando as atividades mencionadas como problemas, a prática de outro exercício físico foi a atividade considerada com maior dificuldade, principalmente pela restrição médica por outras condiçôes de saúde limitantes pela maioria dos idosos. Seguidamente, foram mencionadas as atividades de mobilidade fora de casa, pela dificuldade de deambulação tanto por questóes motoras, quanto pelas barreiras arquitetônicas, interferindo na acessibilidade. Observa-se que a limpeza da casa também é uma atividade mencionada como problema, principalmente pelo gasto energético.

Tabela 1. Características sociodemográficas.

\begin{tabular}{ll}
\hline \multicolumn{1}{c}{ Características } & $\begin{array}{c}\text { Frequência } \\
(\%)\end{array}$ \\
\hline Sexo & \\
Feminino & $34(75,5 \%)$ \\
Masculino & $11(24,4 \%)$ \\
Idade (anos) & \\
$60 \mid-70$ & $16(35,5 \%)$ \\
$70 \mid-80$ & $23(51,1 \%)$ \\
$80 \mid-90$ & $06(13,3 \%)$ \\
Escolaridade & \\
Analfabeto & -- \\
1 a 4 anos de escolarização & $19(42,2 \%)$ \\
5 a 8 anos de escolarização & $13(28,8 \%)$ \\
9 a 11 anos de escolarização & $06(13,3 \%)$ \\
Mais do que 11 anos de & $07(15,5 \%)$ \\
escolarização & \\
Situação Conjugal & \\
Solteiro & $02(4,4 \%)$ \\
Casado & $21(46,6 \%)$ \\
Separado/Divorciado & $04(8,8 \%)$ \\
Viúvo & $17(37,7 \%)$ \\
Outra & $01(2,2 \%)$ \\
Religião & \\
Católica & $35(77,7 \%)$ \\
Evangélica & $05(11,1 \%)$ \\
Espírita & $04(8,8 \%)$ \\
Outra & $01(2,2 \%)$ \\
Ocupação atual & \\
Aposentado & $39(86,6 \%)$ \\
Pensionista & $03(6,6 \%)$ \\
Aposentado ativo & $02(2,2 \%)$ \\
Pensionista ativo & $01(2,2 \%)$ \\
\hline
\end{tabular}

Fonte: Elaborado pelos autores.

Tabela 2. Quantidade de problemas referentes às áreas do desempenho ocupacional.

\begin{tabular}{l|c}
\hline $\begin{array}{c}\text { Áreas do Desempenho } \\
\text { Ocupacional }\end{array}$ & $\begin{array}{c}\text { Problemas no } \\
\text { Desempenho } \\
\text { Ocupacional (\%) }\end{array}$ \\
\hline AUTOCUIDADO & 75 \\
PRODUTIVIDADE & 62,5 \\
LAZER & 80 \\
\hline
\end{tabular}


Tabela 3. Lista de atividades mencionadas como importantes, mas com problemas ou restrições na realização.

\begin{tabular}{|c|c|c|c|}
\hline $\begin{array}{c}\text { Áreas do Desempenho } \\
\text { Ocupacional }\end{array}$ & $\begin{array}{c}\text { Problemas no Desempenho } \\
\text { Ocupacional }\end{array}$ & $\begin{array}{l}\text { Quantidade de } \\
\text { Participantes }\end{array}$ & $\%$ \\
\hline \multicolumn{4}{|c|}{ AUTOCUIDADO } \\
\hline \multirow{2}{*}{ Cuidados Pessoais } & Vestir-se & 11 & 27,5 \\
\hline & Banho & 02 & 5 \\
\hline \multirow{3}{*}{ Mobilidade Funcional } & Mobilidade Fora de Casa & 13 & 32,5 \\
\hline & Transferência & 12 & 30 \\
\hline & Subir/Descer Escadas & 05 & 12,5 \\
\hline \multirow{4}{*}{ Independência Fora de Casa } & Compras & 05 & 12,5 \\
\hline & Finanças & 05 & 12,5 \\
\hline & Dirigir & 02 & 5 \\
\hline & Transporte Público & 01 & 2,5 \\
\hline \multicolumn{4}{|c|}{ PRODUTIVIDADE } \\
\hline \multirow{4}{*}{ Trabalho } & Atividade Voluntária & 05 & 12,5 \\
\hline & Procurar um Trabalho Remunerado & 05 & 12,5 \\
\hline & Manter um Trabalho Remunerado & 03 & 7,5 \\
\hline & Manter um Trabalho Não Remunerado & 01 & 2,5 \\
\hline \multirow{4}{*}{ Tarefas Domésticas } & Limpeza da Casa & 13 & 32,5 \\
\hline & Lavagem de Roupas & 05 & 12,5 \\
\hline & Preparação de Refeições & 01 & 2,5 \\
\hline & Lavar a Louça & 01 & 2,5 \\
\hline Brincar/Escola & - & - & - \\
\hline \multicolumn{4}{|c|}{ LAZER } \\
\hline \multirow{4}{*}{ Recreação Tranquila } & Atividade Manual & 10 & 25 \\
\hline & Leitura & 02 & 5 \\
\hline & Hobbie & 02 & 5 \\
\hline & Tocar um Instrumento Musical & 01 & 2,5 \\
\hline \multirow{2}{*}{ Recreação Ativa } & Praticar Outro Exercício Físico & 23 & 57,5 \\
\hline & Viajar & 09 & 22,5 \\
\hline Socialização & Festas & 02 & 5 \\
\hline
\end{tabular}

Fonte: Elaborado pelos autores.

Percebe-se que, dentre os principais problemas de desempenho ocupacional citados pelos participantes, nove atividades estão relacionadas ao autocuidado, oito à produtividade e sete ao lazer.

A Tabela 4 apresenta uma análise descritiva através das médias e desvio-padrão das áreas do desempenho ocupacional, bem como do desempenho e satisfação das principais atividades-problema dos participantes.

Pelos resultados, percebe-se que as atividades de lazer, por apresentarem maior média, foram consideradas mais importantes pelos idosos praticantes de hidroginástica, principalmente pela prática de exercício físico estar na área de lazer do desempenho ocupacional como uma forma de recreação ativa. Porém, mesmo sendo uma atividade importante, é mencionada como um problema, pois a maioria dos idosos relataram a vontade de praticar também outras modalidades de exercício físico além da hidroginástica. Já a área de produtividade apresenta limitaçóes de desempenho, sinalizadas por uma
Tabela 4. Dados descritivos das médias e desviopadrão das variáveis referente às áreas do desempenho ocupacional, desempenho e satisfação.

\begin{tabular}{lcc}
\hline \multicolumn{3}{c}{ Estatística Descritiva } \\
\hline \multicolumn{1}{c}{ Variáveis } & Média* & Desvio-Padrão \\
\hline Autocuidado & 5,40 & 3,70 \\
Produtividade & 5,00 & 4,19 \\
Lazer & 8,84 & 1,44 \\
Desempenho & 5,38 & 1,92 \\
Satisfação & 6,19 & 2,60 \\
\hline
\end{tabular}

Legenda: *em pontos, considerando escala de 1 a 10 pontos em ordem crescente. Fonte: Elaborado pelos autores.

menor pontuação, principalmente pela dificuldade na atividade de trabalho e também pela dificuldade na realização de tarefas domésticas.

Quanto ao desempenho e a satisfação diante das principais atividades-problema, nota-se que a percepção dos idosos frente ao desempenho é mediana. Mesmo com problemas na realizaçáo das atividades cotidianas, a satisfação com a maneira 
que realizam suas atividades é maior que o próprio desempenho. Na busca de associaçôes entre as variáveis autocuidado e produtividade, autocuidado e lazer, produtividade e lazer, e desempenho e satisfação, obteve-se somente a correlação significativa entre o desempenho e a satisfação $(r=0,770$ e $p \geq 0,001)$.

\section{Discussão}

O presente estudo descreve os principais problemas no desempenho ocupacional de idosos, uma vez que se sabe que o uso da COPM em estudos descritivos oferece um melhor conhecimento e compreensão da profundidade e amplitude das ocupaçóes cotidianas (MCCOLL et al., 2005), ou seja, todos os tipos de atividades diárias importantes para o indivíduo (PERSSON et al., 2014).

A COPM possibilitou investigar as principais atividades cotidianas com problemas no desempenho ocupacional, além de abordar sobre a percepção do indivíduo diante do próprio desempenho, bem como a satisfação na realizaçáo das atividades mencionadas como problema. Assim, além da descrição das atividades, houve a apresentação dos escores médios da COPM, pois mostra melhores resultados do que a reprodutibilidade das pontuaçôes de cada um dos problemas priorizados (EYSSEN et al., 2005).

Sabe-se que a eficiência no desempenho das atividades de vida diária depende de um nível satisfatório de condicionamento físico (PASSOS et al., 2008). Mesmo sabendo dos benefícios da prática regular de exercícios físicos, como a hidroginástica, percebe-se que os idosos ativos também têm dificuldades na realização das atividades cotidianas. Assim, através do uso da COPM, permitiu-se identificar problemáticas no desempenho ocupacional, por meio do apontamento de atividades importantes para o idoso, além de avaliar o desempenho e satisfação em relação às áreas problemáticas (EDWARDS et al., 2007).

A partir da descrição das atividades, no presente estudo houve a identificação de 139 problemas no desempenho ocupacional, sendo que a área do autocuidado apresenta o maior número de problemas frente ao desempenho ocupacional, totalizando 56 problemas. Em um estudo com 50 idosos com fratura de quadril, através do uso da COPM, houve a identificação de 166 problemas de desempenho ocupacional, sendo 119 problemas na área de autocuidado (EDWARDS et al., 2007). Dentro da área do autocuidado, a categoria mencionada com maior dificuldade foi a de mobilidade funcional, com destaque para a atividade fora de casa e, seguidamente, para a transferência. Em estudo realizado com 61 idosos com idades entre 70 e 79 anos, os participantes descreveram 297 limitaçóes de atividade, das quais 228 foram priorizadas, sendo que a atividade mencionada mais vezes como problema foi a mobilidade, principalmente o andar dentro de casa com ou sem andador (TUNTLAND et al., 2015).

A realização de atividades de autocuidado, além de desempenhar um papel social importante quanto ao cuidado de si, ressalta que o indivíduo que é capaz de cuidar de si mesmo, está pronto para ser bem-sucedido na execução de outras tarefas, tais como nas áreas produtivas e de lazer (GARROS; GAGLIARDI; GUZZO, 2010). Considerando as atividades de autocuidado como os principais problemas no desempenho ocupacional, a partir das necessidades expressas pelo indivíduo, salienta-se o investimento de recursos na área de autocuidado (EDWARDS et al., 2007), uma vez que o indivíduo tende a escolher as tarefas relacionadas à sobrevivência quando questionado sobre suas necessidades na intenção de tornar-se menos dependente de outras pessoas (GARROS; GAGLIARDI; GUZZO, 2010).

Quanto às outras atividades mencionadas como problema, o vestir-se como atividade de cuidados pessoais da área do autocuidado e a limpeza da casa como tarefa doméstica da área da produtividade também são consideradas importantes para os idosos do estudo. O que é verificado em outro estudo com idosos, referindo também dificuldades nas tarefas domésticas, ressaltando-se a limpeza da casa, e dos cuidados pessoais, destacando-se o vestir-se (TUNTLAND et al., 2015). Outros estudos (BECKER; MONTILHA, 2015; CUP et al., 2003; GARROS; GAGLIARDI; GUZZO, 2010), usando a COPM, também identificaram problemas no desempenho ocupacional, principalmente nas atividades na área de autocuidado.

No presente estudo, houve a identificaçáo de problemas no desempenho em atividades da área do lazer, destacando-se a prática de outro exercício físico, sendo uma recreação ativa, por ser a atividade com maior dificuldade de realizaçáo dos idosos. Considerando as médias de importância, percebe-se que as atividades de lazer são mais importantes, mesmo apresentando dificuldades na realização. Além da prática de outro exercício físico, as atividades manuais também foram consideradas uma importante recreação tranquila, apesar das dificuldades na realização. Um estudo utilizando a COPM com 326 idosos identificou que idade superior a 70 anos, incapacidade cognitiva e doenças crônicas como asma, hipertensão e diabetes são fatores de risco para perda da capacidade funcional de idosos, mas 
que realizar um trabalho remunerado, atividades de lazer como assistir televisão e realizar atividades manuais, e o suporte social como o relacionamento mensal com amigos, podem ser considerados fatores de proteção ao idoso (D'ORSI; XAVIER; RAMOS, 2011). Assim, pode-se argumentar sobre a importância das atividades de lazer, como as atividades de socialização, bem como de recreação tranquilas e ativas para a população idosa, ressaltando-se a prática de hidroginástica também permite a integração com outros participantes do grupo, minimizando a questão do isolamento social. Uma das principais formas de participação em grupo e de visualização social dos idosos são as atividades culturais e de lazer, como: grupos de convivência, clubes da "maior" idade e programas ou cursos para a "terceira idade" (MOTTA, 1999, p. 219). Além de que um estilo de vida ativo promove a manutenção da capacidade funcional dos idosos por um período mais longo e, consequentemente, mantém a qualidade de vida, sendo essencial "[...] modos de viver mais saudáveis em todas as etapas da vida, favorecendo a prática de atividades físicas no cotidiano e no lazer [...]" (ORGANIZAÇĀO..., 2005, p. 3).

Estudos com diferentes populaçōes (EDWARDS et al., 2007; GARROS; GAGLIARDI; GUZZO, 2010; NICKEL et al., 2012) também identificaram problemas na produtividade e no lazer. $\mathrm{Na}$ área de produtividade, atividades como procurar ou manter um trabalho remunerado, ou até mesmo atividade voluntária, foram consideradas importantes para os idosos, mas sendo também uma atividade-problema, podendo-se justificar pela alteração nos papéis sociais, principalmente pela aposentadoria. Sabe-se que o trabalho remunerado tem efeito protetor contra a incapacidade funcional de idosos, através de mecanismos de suporte social pelo convívio com outras pessoas (D'ORSI; XAVIER; RAMOS, 2011). Mesmo que as atividades relacionadas ao lazer não sejam frequentemente citadas como problemas no desempenho ocupacional, a área do lazer é extremamente importante, pois o indivíduo, principalmente aquele com alguma incapacidade, pode estar inserido em uma posição abaixo do nível de outros indivíduos em competição social, necessitando de um estímulo para resgatar ou iniciar atividades de lazer (GARROS; GAGLIARDI; GUZZO, 2010).

Além da descrição das atividades com problemas no desempenho, bem como a percepção do desempenho e da satisfação, o presente estudo apresentou uma correlação significativa entre o desempenho e a satisfação. A correlação significativa entre as duas variáveis pode-se justificar considerando que, à medida que o resultado na realização das atividades cotidianas acontece, também ocorre a satisfação pessoal em relação a este desempenho. Assim, sugere-se que quanto melhor o desempenho na realização das atividades cotidianas, melhor a satisfação do idoso, uma vez que mudanças no desempenho e na satisfaçâo com o desempenho ocupacional estâo associadas a mudanças no funcionamento psicossocial e bem-estar psicológico (PERSSON et al., 2014). Há outra questão referente à satisfação, afirmando que o processo de entrevista e pontuação da COPM pode ter um efeito terapêutico, podendo promover a consciência e motivação (LANGELAND et al., 2015), melhorando assim a percepção em relação à satisfação no desempenho ocupacional. Em contrapartida, a relação do desempenho ocupacional com uma baixa percepção de satisfação pode-se justificar pela perda da motivação para se manter ativo e independente sobre suas próprias vidas, reduzindo a autoestima (BECKER; MONTILHA, 2015).

Um melhor desempenho e, consequentemente, uma melhor satisfação na realização das atividades cotidianas sugerem que o efeito de um programa de exercícios, que incide sobre a melhora da força, da resistência e do equilíbrio, é significativo para os indivíduos e também tem um impacto relevante sobre o desempenho e satisfação, além de estar relacionados com a melhoria da saúde e da qualidade de vida (ENG et al., 2003). Cabe ressaltar também a busca da prática da hidroginástica como modalidade preventiva, uma vez que existe uma indicação que, através das atividades de prevenção, existe uma maior probabilidade de melhorar o desempenho ocupacional e a satisfação (PERSSON et al., 2014).

A pontuaçáo do desempenho e da satisfação do indivíduo deve ser medida com a proposta de se reavaliar e comparar os domínios para, assim, comprovar a eficácia do tratamento (LAW et al., 2009), pois um aspecto positivo do uso da COPM é a possibilidade de desenvolver programas individualizados a partir da identificação de atividades-problema e de detectar mudanças no desempenho e na satisfação, após um período de intervençáo (CARSWELL et al., 2004). Porém, como não houve a reavaliação do desempenho e da satisfação, não foi possível identificar mudanças na percepção do idoso frente ao desempenho ocupacional, sendo que a COPM tem fortes propriedades psicométricas no teste-reteste, tanto para a pontuação do desempenho quanto para a satisfação (CARSWELL et al., 2004; LAW et al., 2009). Assim, pode-se pensar a possibilidade de reavaliação do desempenho e da satisfação, sendo uma sugestão de continuidade do estudo, inserindo a COPM na avaliação de intervençôes de programas, como medida de resultado, inclusive 
na prática da hidroginástica, com a finalidade de estabelecer metas e prioridades para um grupo e fornecer informaçóes de planejamento e definiçáo de tratamento (MCCOLL et al., 2005).

\section{Conclusão}

O uso da COPM permitiu identificar os principais problemas no desempenho ocupacional de idosos praticantes de hidroginástica. Mesmo apresentando um resultado significativo na correlação do desempenho com a satisfação, o estudo apresenta duas limitações. Primeiramente, não houve uma proposta de afirmar a eficácia de um método de intervenção, mas apenas descrever os principais problemas de desempenho de uma população de idosos praticantes de hidroginástica. Assim, houve uma dificuldade na busca de estudos para fomentar a discussão, podendo apresentar somente aqueles com descrição dos problemas. Outra limitação é a escassez de estudos que utilizam a COPM com a população idosa ativa, bem como na prática clínica com a intervenção por meio do exercício físico, não sendo possível comparar os dados descritivos do estudo com investigaçôes similares por não serem encontradas na literatura.

A partir das principais atividades-problema e da percepção do idoso sobre seu desempenho e sua satisfação diante das atividades cotidianas, existe a possibilidade de desenvolver programas individualizados de intervenção, ou até mesmo em grupo, na tentativa de verificar mudanças no desempenho ocupacional com a prática de hidroginástica e também com outras modalidades. Considera-se, então, importante a execução de projetos com outras propostas de exercícios físicos, uma vez que são consideradas atividades importantes para os idosos, de acordo com suas necessidades e condiçôes de saúde. Além de saber que a prática de exercícios físicos é uma forma de contribuir para um envelhecimento ativo e com qualidade de vida na velhice, podendo também refletir no desempenho positivo de atividades das áreas de autocuidado, como a mobilidade funcional, e de produtividade, como a realizaçáo de tarefas domésticas.

Assim, sugere-se a continuidade no estudo, na tentativa de realizar a reavaliaçáo do desempenho ocupacional e da satisfação dos idosos participantes do estudo, inclusive na consideração de outras modalidades de exercício físico. Observa-se, também, a necessidade de novas pesquisas para comparação dos dados, já que não foram encontrados outros estudos envolvendo o uso da COPM com idosos praticantes de hidroginástica.

\section{Referências}

BECKER, P.; MONTILHA, R. C. Ocupational performance and quality of life: interrelationships in daily life of visual impaired individuals. Revista Brasileira de Oftalmologia, Rio de Janeiro, v. 74, n. 6, p. 372-377, 2015.

BRASIL. Ministério da Saúde. Resolução no 466, de 12 de Dezembro de 2012. Diário Oficial [da] República Federativa do Brasil, Poder Executivo, Brasília, DF, 2012.

BRUCKI, S. M. D. et al. Sugestấo para o uso do Mini-Exame do Estado Mental no Brasil. Arquivos de Neuro-Psiquiatria, São Paulo, v. 61, n. 3B, p. 777-781, 2003.

CARSWELL, A. et al. The Canadian Occupational Performance Measure: a research and clinical literature review. Canadian Journal of Occupational Therapy, Ottawa, v. 71, n. 4, p. 210-222, 2004.

CUP, E. H. et al. Reliability and validity of the Canadian Occupational Performance Measure in stroke patients. Clinical Rehabilitation, London, v. 17, n. 4, p. 402-409, 2003.

D'ORSI, E.; XAVIER, A. J.; RAMOS, L. R. Trabalho, suporte social e lazer protegem idosos da perda funcional: estudo epidoso. Revista de Saúde Pública, São Paulo, v. 45, n. 4, p. 685-692, 2011.

EDWARDS, M. et al. Recovery after hip fracture: what can we learn from the Canadian Occupational Performance Measure? American Journal of Occupational Therapy, Rockville, v. 61, n. 3, p. 335-344, 2007.

ENG, J. J. et al. A community-based group exercise program for persons with chronic stroke. Medicine \& Science in Sports \& Exercis, Indianapolis, v. 35, n. 8, p. 1271-1278, 2003.

EYSSEN, I. C. et al. The reproducibility of the Canadian Occupational Performance Measure. Clinical Rehabilitation, London, v. 19, n. 8, p. 888-894, 2005.

FECHINE, B. R. A. O.; TROMPIERI, N. Processo de envelhecimento: as principais alteraçóes que acontecem com o idoso com o passar dos anos. InterScience Place, Campos dos Goytacazes, v. 1, n. 20, p. 115-121, 2012.

GARROS, D. S. C.; GAGLIARDI, R. J.; GUZZO, R. A. R. Evaluation of performance and personal satisfaction of the patient with spastich and after using a volar dorsal orthosis. Arquivos de Neuro-Psiquiatria, São Paulo, v. 68, n. 3, p. 385-389, 2010.

LANGELAND, E. et al. I. Study protocol for a multicenter investigation of reablement in Norway. BMC Geriatrics, London, v. 15, p. 1-9, 2015. http://dx.doi. org/10.1186/s12877-015-0108-y.

LAW, M. et al. The Canadian Occupational performance measure: an outcome measure for Occupational Therapy. Canadian Journal of Occupational Therapy, Ottawa, v. 57, n. 2, p. 82-87, 1990.

LAW, M. et al. Medida Canadense de Desempenho Ocupacional (COPM). Belo Horizonte: Editora Universidade Federal de Minas Gerais, 2009. 
MAZO, G. Z.; LOPES, M. A.; BENEDETTI, T. B. Atividade física e o idoso: concepção gerontológica. Porto Alegre: Sulina, 2004.

MCCOLL, M. A. et al. Targeted applications of the Canadian Occupational Performance Measure. Canadian Journal of Occupational Therapy, Ottawa, v. 72, n. 5, p. 298-300, 2005.

MOTTA, A. B. As dimensóes de gênero e classe social na análise do envelhecimento. Cadernos Pagu, Campinas, n. 13, p. 191-221, 1999.

NICKEL, R. et al. Quality of life issues and occupational performance of persons with epilepsy. Arquivos de Neuro-Psiquiatria, São Paulo, v. 70, n. 2, p. 140-144, 2012.

ORGANIZAÇÃO MUNDIAL DA SAÚDE - OMS. Envelhecimento ativo: uma política de saúde. Brasília: OMS, 2005.

PASSOS, B. M. A. et al. Contribuiçôes da hidroginástica nas atividades da vida diária na flexibilidade de mulheres idosas. Revista da Educação Física, Maringá, v. 19, n. 1, p. 71-76, 2008.
PEDRETTI, L. W.; EARLY, M. B. Desempenho Ocupacional e Modelos de Prática para Disfunção Física. In: PEDRETTI, L. W.; EARLY, M. B. Terapia Ocupacional: capacidades práticas para disfunçóes físicas. São Paulo: Roca, 2004. p. 3-13.

PERSSON, E. et al. Occupational performance and factors associated with outcomes in patients participating in a musculoskeletal pain rehabilitation programme. Journal of Rehabilitation Medicine, Uppsala, v. 46, n. 6, p. 546-552, 2014.

SILVA, A. G.; RIBEIRO, J. C. Hidroginástica na terceira idade. Ágora: Revista de Divulgação Científica, Mafra, v. 17 , n. 2, p. 49-59, 2010

THOMAS, J. R.; NELSON, J. K.; SILVERMAN, S. J. Métodos de pesquisa em atividade física. Porto Alegre: Artmed, 2012.

TUNTLAND, H. et al. Reablement in community-dwelling older adults: a randomised controlled trial. BMC Geriatrics, London, v. 15, p. 1-11, 2015.

ZAGO, A. S. Exercício físico e o processo saúde-doença no envelhecimento. Revista Brasileira de Geriatria e Gerontologia, Rio de Janeiro, v. 13, n. 1, p. 153-158, 2010.

\section{Contribuição dos Autores}

Kátine Marchezan Estivalet foi responsável pela concepção e redação do presente estudo. Sara Teresinha Corazza orientou o estudo. Todos os autores aprovaram a versão final do texto. 